\title{
Ordering of the lamellar phase under a shear flow
}

\author{
F. Corberi \\ Istituto Nazionale per la Fisica della Materia, Unità di Salerno and Dipartimento di Fisica, Università di Salerno, 84081 \\ Baronissi (Salerno), Italy \\ G. Gonnella ${ }^{(+)}$and A. Lamura ${ }^{(+)(*)}$ \\ (+) Istituto Nazionale per la Fisica della Materia and Dipartimento di Fisica, Università di Bari and Istituto Nazionale di \\ Fisica Nucleare, Sezione di Bari, via Amendola 173, 70126 Bari, Italy \\ (*) Istituto Applicazioni Calcolo, CNR, Sezione di Bari, via Amendola 122/I - 70126 Bari - Italy
}

(October 24, 2018)

The dynamics of a system quenched into a state with lamellar order and subject to an uniform shear flow is solved in the large- $N$ limit. The description is based on the Brazovskii free-energy and the evolution follows a convection-diffusion equation. Lamellae order preferentially with the normal along the vorticity direction. Typical lengths grow as $\gamma t^{5 / 4}$ (with logarithmic corrections) in the flow direction and logarithmically in the shear direction. Dynamical scaling holds in the two-dimensional case while it is violated in $D=3$.

PACS numbers: 64.75.+g; 05.70.Ln; 47.20.Hw

Many systems in nature exhibit lamellar order. One example is a diblock copolymer melt where chains of type A and B covalently bonded end-to-end in pairs segregate at low temperatures with A-B junctions forming a stack of lamellae [1]. In ternary mixtures a lamellar phase is stable with ordered sheets of surfactant separating (say) oil and water domains [2]. Lamellar order is also observed in Raleigh-Bénard cells, where convective rolls form above the convective threshold [3]. Further examples include smectic liquid crystals [4], dipolar fluids with long-range interactions $[5$ and chemically reactive binary mixtures [6]. A theoretical model for the general description of the lamellar-disordered phase transition was proposed by Brazovskii [7] who showed the first-order character of the transition induced by fluctuations.

Lamellar phases under an applied shear flow show a very rich behavior which is relevant for many applications [8]. A variety of transitions in morphology and orientation occur as shear rate and temperature are changed. Stable configurations of lamellae lying along the flow with the normals differently oriented have been observed 9.10] and analyzed evaluating the effects of the flow on the fluctuation spectra [11, 12, 13, 14].

Non-stationary properties are far less considered [15, 16, 17, 18]. In this Letter we study the effects of a simple planar shear flow on the ordering of the lamellar phase in a system quenched from an initially high temperature disordered state. We present the first analytical results on the kinetics of this system by solving the Brazovskii model in the limit of an infinite number of components of the order parameter. This is one of the few methods allowing an explicit solution for phase ordering systems [19]. A similar approach for fluids under shear flow has been used in $20,21,22,23,24,25,26,27]$.

The behavior of quenched binary mixtures without imposed flows is characterized by dynamical scaling: The structure factor obeys $C(\vec{k}, t)=R(t)^{D} f[k R(t)]$ where $R(t) \sim t^{\alpha}$ is the typical domain size and $D$ is the space dimensionality [28, 19]. In the case of a fluid with lamellar order, if the order parameter is not conserved as in the Swift-Hohenberg model for Raleigh-Bénard convection [29], regimes exhibiting dynamical scaling have been found 30]. In models with conserved order parameter, when diffusion is the only segregating physical mechanism, simulations show the entanglement of the fluid into frozen intertwined structures [31,32]. In this case the effects of hydrodynamical modes are crucial for reaching order on large scales [33].

When shear is applied, our results show that lamellae grow preferentially with the perpendicular orientation [12], namely along the plane formed by the flow and the shear (velocity gradient) directions. Their typical size, obtained from the second momentum of the structure factor, grows as $\gamma t^{5 / 4} \sqrt{\ln t}$ in the flow direction and as $\sqrt{\ln t}$ in the shear direction. Surprisingly we find that dynamical scaling is obeyed in two dimensions but not in $D=3$. Our results concern the cases of conserved and not conserved order parameter, and apply to most of the systems mentioned above.

We consider the Brazovskii free energy

$$
\mathcal{F}\{\phi\}=\int d \vec{r}\left\{\frac{r}{2} \phi^{2}+\frac{u}{4} \phi^{4}-\frac{b}{2}|\nabla \phi|^{2}+\frac{1}{2}\left(\nabla^{2} \phi\right)^{2}\right\}
$$

where $\phi(\vec{r}, t)$ is the order parameter field and $u>0$. With a negative value of $r$ and $b<0$ the system orders in one of the two minima of the local potential. However, when $b>0$ interfaces are favoured and a modulated state with wavevector $k_{M}=\sqrt{b / 2}$ is stable [7].

When a flow is imposed, the kinetics can be described by the convection-diffusion equation [34]

$$
\frac{\partial \phi}{\partial t}+\vec{\nabla} \cdot(\phi \vec{v})=-\Gamma_{p} \frac{\delta \mathcal{F}}{\delta \phi} \quad, \quad p=0,2
$$

where 


$$
\Gamma_{0}=\Gamma, \quad \Gamma_{2}=-\Gamma \nabla^{2}
$$

and $\Gamma$ is a mobility coefficient. $p=0$ describes systems with non-conserved order parameter (NCOP) and corresponds to the Swift-Hohenberg equation [29]; the case $p=2$ is for conserved order parameter $(\mathrm{COP})$ and applies for example to copolymer melts. For uniform shear flow in the $x$-direction $v_{x}=\gamma y, \gamma$ being the shear rate. Eq.(2) neglects thermal fluctuations and possible effects due to differences in viscosities between the two components 12. The complete description should take into account the coupling of Eq. (2) with the Navier-Stokes equation. However, the study of Eq. (2) is a prerequisite for any more general theory and usually applies to intermediate temporal regimes, as recognized for phase separation of simple binary mixtures [19].

Eq. (2) can be studied analytically generalizing the field $\phi$ to a vector order parameter with $N$ components and taking the large- $N$ limit [35]. One obtains the following equation in Fourier space for the structure factor $C(\vec{k}, t)=\langle\phi(\vec{k}, t) \phi(-\vec{k}, t)\rangle$

$$
\begin{aligned}
\frac{\partial C(\vec{k}, t)}{\partial t} & -\gamma k_{x} \frac{\partial C(\vec{k}, t)}{\partial k_{y}}= \\
& -2 \Gamma k^{p}\left[r+u S(t)-b k^{2}+k^{4}\right] C(\vec{k}, t)
\end{aligned}
$$

where we have dropped the component indices due to internal symmetry. $S(t)$ has to be computed selfconsistently through

$$
S(t)=\int_{|k|<\Lambda} \frac{d \vec{k}}{(2 \pi)^{D}} C(\vec{k}, t),
$$

$\Lambda$ being an ultraviolet cut-off. For a symmetric mixture quenched from a high temperature homogeneous phase an appropriate initial condition is $C(\vec{k}, 0)=\Delta$, where $\Delta$ is a constant. Eq. (4) can be integrated yielding

$$
C(\vec{k}, t)=\Delta e^{-2 \Gamma \int_{0}^{t} d \tau \overrightarrow{\mathcal{K}}^{p}(\tau)\left[\mathcal{K}^{4}(\tau)-b \mathcal{K}^{2}(\tau)+r+u S(t-\tau)\right]}
$$

where $\overrightarrow{\mathcal{K}}(\tau) \equiv \vec{k}+\gamma \tau k_{x} \vec{e}_{y}$. Defining

$$
Q(t) \equiv \int_{0}^{t} d \tau[r+u S(t-\tau)]
$$

the analysis can be carried out asymptotically through the ansatz

$$
Q(t)=k_{M}^{4} t-\frac{1}{2 \Gamma k_{M}^{p}}\left(2 \ln t-\ln \ln t+\ln v_{p}\right),
$$

where $v_{p}$ is a constant to be determined. Eq.(8) will be justified a posteriori by proving the solution of the selfconsistency problem. Specifically, the large time value $S_{\infty}$ calculated through (5), inserted into (7), must satisfy (8) asymptotically, namely

$$
S_{\infty}=\left(k_{M}^{4}-r\right) / u
$$

We now briefly illustrate this for COP in $D=3$ 36 setting $\Gamma=1=u=b=-r$.

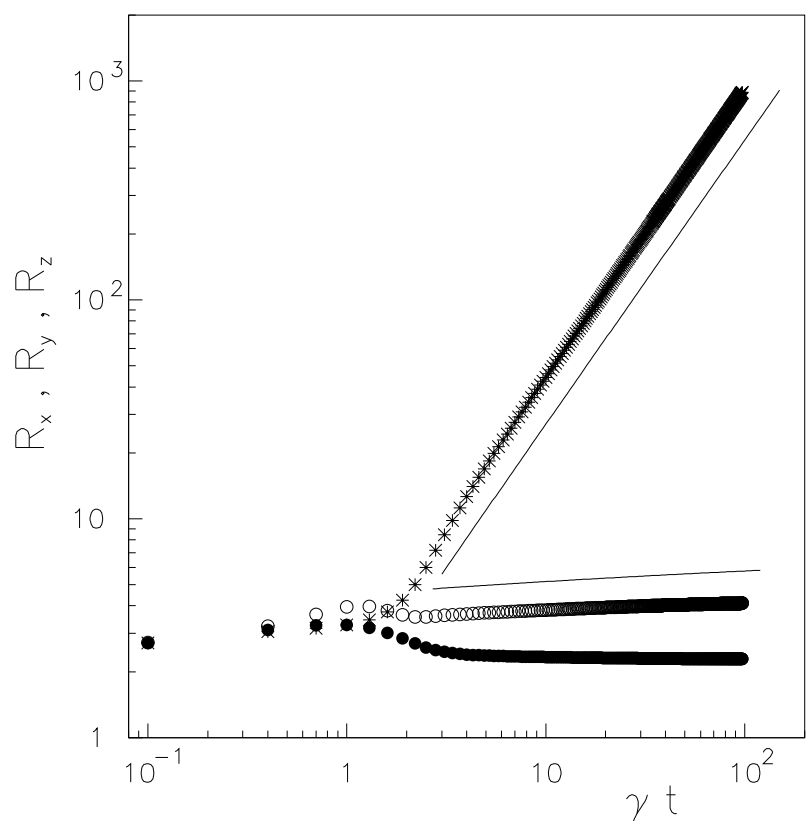

FIG. 1. The typical lengths as a function of the shear strain in the $x(*), y(\circ)$ and $z(\bullet)$ directions. The lines are proportional to $\gamma t^{5 / 4}(\log t)^{1 / 2}$ and $(\log t)^{1 / 2}$.

The symmetry of the problem suggests the use of the cylindrical set of variables $\left(k_{x}, k_{\perp}, \theta\right)$, where $k_{y}=$ $k_{\perp} \cos \theta$ and $k_{z}=k_{\perp} \sin \theta$. Naive power counting applied to Eq. (6) indicates that wave vectors in the flow direction scale asymptotically as $k_{x} \sim t^{-3 / 2}$ while in the perpendicular directions $k_{\perp}-k_{M} \sim t^{-1 / 2}$. Then, introducing the new variables $X=k_{x} L_{\|}(t), Q=\left(k_{\perp}-k_{M}\right) L_{\perp}(t)$, with $L_{\|}(t)=2 \sqrt{2 / 3} k_{M}^{2} \gamma t^{3 / 2}$ and $L_{\perp}(t)=2 \sqrt{2 t} k_{M}^{2}$, and taking into account Eq. (8), Eq. (6) reads as

$$
C(\vec{k}, t)=v_{2} \Delta \frac{t^{2}}{\ln t} e^{-[f(X, Q, \theta)+g(X, Q, \theta, t)]}
$$

with

$$
f=Q^{2}+\sqrt{3} Q X \cos \theta+X^{2} \cos ^{2} \theta
$$

and $g(X, Q, \theta, t)=\sum_{j=1}^{16} t^{-j / 2} g_{j}$, where $g_{j}$ are polynomials in $X, Q, \theta, \log t$. For long times $g$ can be shown to provide a relevant contribution $g_{2} \simeq \frac{9}{160} \frac{X^{4}}{k_{M}^{6}}-\frac{3}{4 k_{M}^{6}} X^{2} \ln t$ only in the region $Q \sim 0, \cos \theta \sim 0$. Then the integration over $Q$ and $\theta$ can be performed yielding

$$
S(t) \simeq \frac{\sqrt{3} \Delta}{32 k_{M}^{3} \pi^{\frac{3}{2}}} \frac{v_{2}}{\gamma \ln t} \int_{-\infty}^{\infty} d X e^{-\frac{X^{2}}{8}-\frac{g_{2}}{t}} I_{0}\left(X^{2} / 8\right)
$$

where $I_{0}(z)$ is a Bessel function. The integral of Eq. (12), evaluated by the asymptotic expansion of $I_{0}(z)$ at large $z$, behaves as $\ln t / \sqrt{\pi}$. Hence at large times $S(t)$ approaches the constant $S_{\infty}=\frac{\sqrt{3} v_{2} \Delta}{32 \pi^{2} k_{M}^{3} \gamma}$. A comparison with Eq.(9) fixes $v_{2}=\frac{32 \pi^{2} k_{M}^{3} \gamma\left(k_{M}^{4}+1\right)}{\sqrt{3} \Delta}$ and verifies the ansazt. 
Due to the presence of $g$, the function $C(\vec{k}, t)$ of Eq.(10) cannot be cast in a scaling form. However, different scaling behaviors are obeyed in the regions where the functions $f$ or $g$ can be respectively neglected. In particular, for $Q \sim 0, \cos \theta \sim 0$, where $g$ dominates, $C(\vec{k}, t)$ scales in the $x$-direction with respect to the length $l_{\|}(t)=(2 / 5)^{1 / 4} \sqrt{k_{M}} \gamma t^{5 / 4}$ different from $L_{\|}(t)$. In $D=2$, on the other hand, where the contribution of the function $g$ is always negligible, one finds the scaling form $C(\vec{k}, t)=v_{2} \Delta t^{2} e^{-[f(X, Q, 0)]}$.
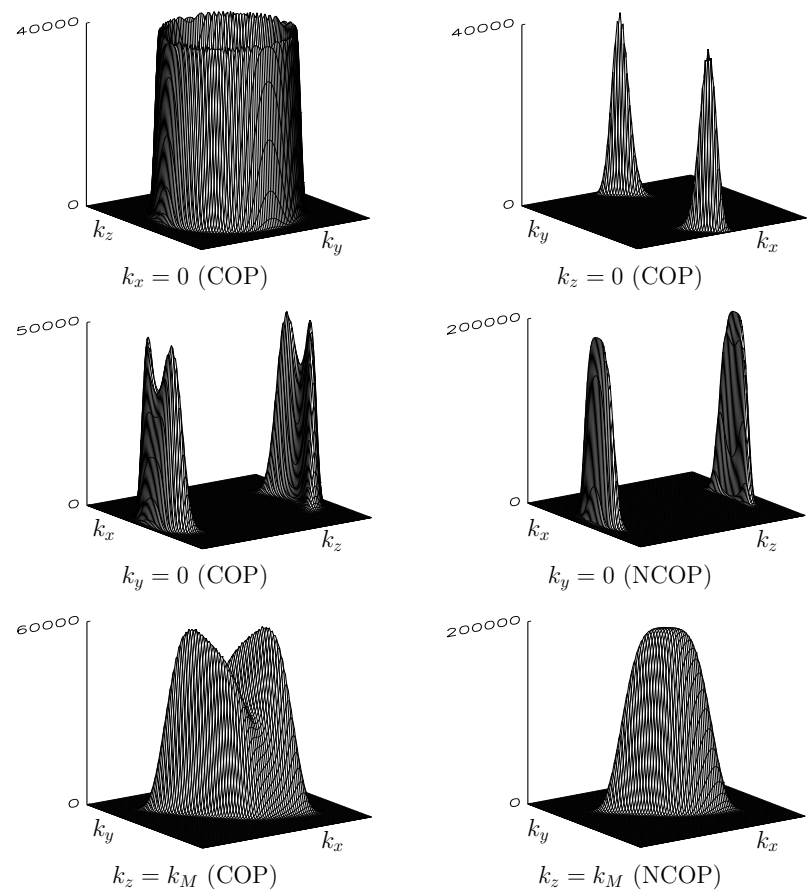

FIG. 2. The structure factor is shown for the COP case on the planes $k_{x}=0, k_{y}=0, k_{z}=0, k_{z}=k_{M}$ and for the NCOP case on the planes $k_{y}=0, k_{z}=k_{M}$.

Ordering properties are usually inferred from the momenta of the structure factor. We define $R_{x}=$ $\left[\int d \vec{k} C(\vec{k}, t) / \int d \vec{k} k_{x}^{2} C(\vec{k}, t)\right]^{1 / 2}$ and similarly for the shear and vorticity directions. In the absence of dynamical scaling definitions of $R_{x}$ based on different momenta of $C(\vec{k}, t)$ may lead to different results. However in this case it can be proven that changing the order of the momentum does not change the growth exponent but only the logarithmic correction. ¿From Eq. (10) we find $R_{x} \sim \gamma t^{5 / 4} \sqrt{\ln t}, R_{y} \sim \sqrt{\ln t}$ and $R_{z} \sim k_{M}^{-1}$. The behavior of $R_{x}$ shows the relevance of the scaling with respect to $l_{\|}(t)$ for the ordering of the system. The growth of $R_{y}$ indicates that lamellae order preferentially in the plane $x-y$. In $D=2$ we find $R_{x} \sim \gamma t^{3 / 2}$ and $R_{y} \sim k_{M}^{-1}$. The same results are obtained with non-conserved order parameter. The behavior of $R_{x}, R_{y}, R_{z}$ resulting from the numerical integration of Eq. (4) with an adaptive grid algorithm is shown in Fig. 1. After the initial isotropic evolution, the shear-induced anisotropy becomes evident for values of the strain $\gamma t$ larger than one and agreement with the analytical behavior is observed.

It is also useful to illustrate the behavior of the structure factor (10) shown in Fig. 2. The maxima of $C(\vec{k}, t)$ are located at $k_{x}^{2}=5 \frac{1}{k_{M}^{4} \gamma^{2}} \frac{\ln t}{t^{3}}, k_{y}=0, k_{z}^{2}=k_{M}^{2}$ for COP and at $k_{x}=0, k_{y}^{2}+k_{z}^{2}=k_{M}^{2}$ for NCOP. The shape of $C(\vec{k}, t)$ on the planes $k_{x}=0$ and $k_{z}=0$ is qualitatively similar for COP and NCOP. At $k_{x}=0$, in particular, the wavevectors are not affected by the flow and the structure factor has a circular structure with radius $k_{M}$. At $k_{y}=0$ or $k_{z}=k_{M}$ the behavior of $C(\vec{k}, t)$ depends on the conservation law. The maxima of $C(\vec{k}, t)$ developed with NCOP are splitted with COP into a pair of symmetric narrowing peaks. This pattern is typical of the case with conserved dynamics and has been observed in other segregating systems under shear flow [37].

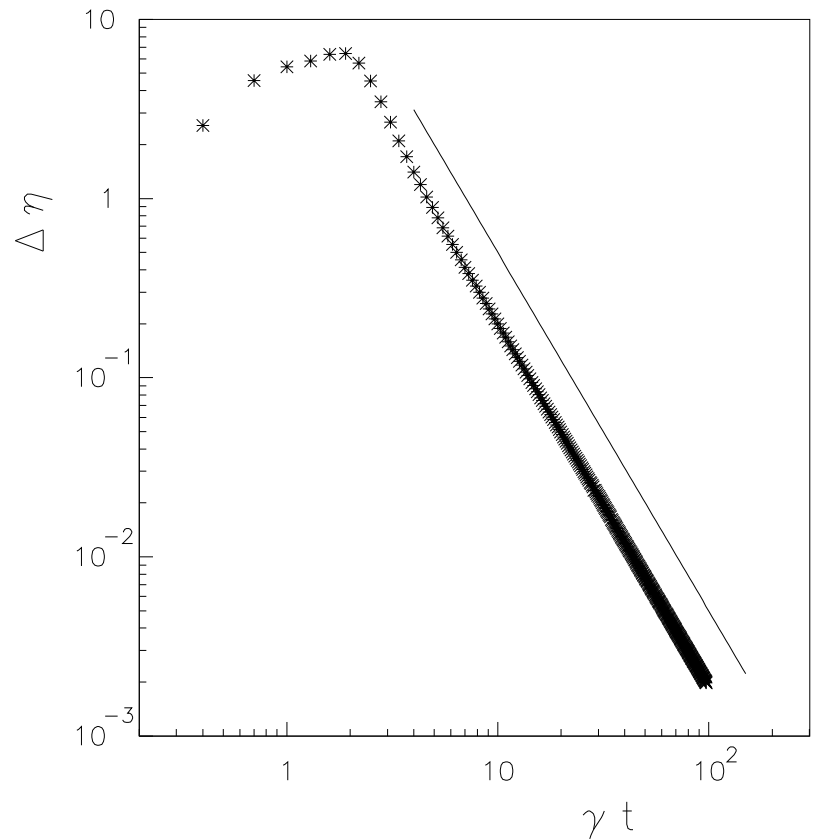

FIG. 3. The excess viscosity as a function of the shear strain. The straight line has slope -2 .

Finally we turn to the study of the rheological properties. In phase separation of binary mixtures the flow acts against the surface tension inducing stretching of domains followed by break-up processes and burst of small bubbles [38,39]. An excess viscosity $\Delta \eta$ is measured [40]: it reaches a maximum generally for $\gamma t \geq 1$ and later decays. In our case we measure the evolution of the shear stress $\sigma_{x y}$ deducible in a general way from (11) in terms of the structure factor; it is given by $\sigma_{x y}(t)=\int \frac{d \vec{k}}{(2 \pi)^{D}} k_{x} k_{y}\left(2 k^{2}-b\right) C(\vec{k}, t)$ [24]. An excess viscosity can be introduced as $\Delta \eta(t)=-\sigma_{x y}(t) / \gamma$ [11]. Using Eq. (10) we find $\Delta \eta \sim \gamma^{-1} t^{-2}$. The numerical results of Fig. 3 show that, after reaching a maximum, the decay of $\Delta \eta$ agrees with the analytical behavior. The other 
rheological indicators can be shown to behave similarly.

In conclusion we have studied the ordering kinetics of a lamellar phase in shear flow by solving the dynamics of the convective-diffusion equation in the large-N limit. Regarding the debated question of the stable orientation, our results are in agreement with the expectation of the stability of the perpendicular phase at high shear rates [12]. In $D=3$ a logarithmic growth law is found along the shear direction while $R_{x} \sim \gamma t^{5 / 4} \sqrt{\ln t}$. Interestingly, the same exponent $5 / 4$ is found for simple binary fluids 26,27]. Our results show that the scaling depends on dimensionality and is violated in $D=3$. This is at variance with the case without shear where the same approach gives a scaling form independently of $D$ [42]. Violation of scaling is quite uncommon [43,19]. In this context this phenomenon may be related to the existence of two lengths $L_{\|}, l_{\|}$growing with different exponents.

A natural question is the relevance of the behavior of the large-N model to the physical case with $N=1$. As without flow, symmetry arguments suggest an analogy between the coarsening behavior of lamellar phases and that of vectorial models and one would argue that the large- $\mathrm{N}$ results give reliable indications also for the physical case [44]; in particular the large- $\mathrm{N}$ exponent is the one expected asymptotically [30]. Simulations of the scalar case with shear could elucidate this point; however strong finite size effects make the evaluation of the growth exponent difficult (see [39] and refs. therein). We also mention that it will be important to study how the presence of hydrodynamics affects the picture provided in this Letter.

We thank Mario Pellicoro and Marco Zannetti for useful discussions. F.C. and G.G. acknowledge M.Cirillo and R. Del Sole for hospitality in Rome University and support by the PRA-HOP 1999 INFM and MURST (PRIN 1999).

[1] S.F. Bates, Science 251, 898 (1991).

[2] G. Gompper and M. Schick, Phase transitions and critical phenomena vol. 16, eds. C. Domb and J.L. Lebowitz, (Academic Press, New York, 1994).

[3] M. C. Cross and P. C. Hohenberg, Rev. Mod. Phys. 65, 851 (1993).

[4] See, for example, P. de Gennes, The Physics of Liquid Crystals (Clarendon, Oxford, 1974).

[5] C. Roland and R. C. Desai, Phys. Rev. B 42, 6658 (1990); C. Sagui and R. Desai, Phys. Rev. Lett. 71, 3995 (1993).

[6] S. C. Glotzer, E. A. Di Marzio, and M. Muthukumar, Polym. Mater. Sci. Eng. Proc. Am. Chem. Soc. 71, 645 (1994); S. C. Glotzer, D. Stauffer, and N. Yan, Phys. Rev. Lett. 72, 4109 (1994).

[7] S. A. Brazovskii, Sov. Phys. JETP 41, 85 (1975).
[8] See, e.g., R. G. Larson, The structure and Rheology of Complex fluids (Oxford University Press, New York, 1999).

[9] K. A Koppi, M. Tirrell, F. S. Bates, K. Almdal, and R. H. Colby, J. Phys. France 2, 1941 (1992).

[10] K. I. Winey, S. S. Patel, R. G. Larson, and H. Watanabe, Macromolecules 26, 2542 (1993).

[11] M. E. Cates and S. T. Milner, Phys. Rev. Lett. 62, 1856 (1989).

[12] G. Fredrickson, J. Rheol. 38, 1045 (1994).

[13] M. Goulian and S. T. Milner, Phys. Rev. Lett. 74, 1775 (1995).

[14] A. N. Morozov, A. V. Zvelindovsky, and J. G. E. M. Frajie, preprint cond-mat/0104339.

[15] H. Kodama and M. Doi, Macromolecules 29, 2652 (1996).

[16] M. Bahiana, Physica A 257, 307 (1998).

[17] F. Drolet, P. Chen, and J. Viñals, Macromolecules 32, 8603 (1999).

[18] N. A. Gross, M. Ignatiev, and B. Chakraborty, Phys. Rev. E 62, 6116 (2000).

[19] See, e.g., A. J. Bray, Adv. Phys. 43, 357 (1994).

[20] A. Onuki and K. Kawasaki, Ann. Phys. (N.Y.) 121, 456 (1979).

[21] G. H. Fredrickson, J. Chem. Phys. 85, 5306 (1986).

[22] G. H. Fredrickson and R. G. Larson, J. Chem. Phys. 86, 1553 (1987).

[23] A. Onuki, J. Chem. Phys. 87, 3692 (1987).

[24] G. Pätzold and K. Dawson, Phys. Rev. E 54, 1669 (1996). G. Pätzold and K. Dawson, J. Chem. Phys. 104, 5932 (1996).

[25] C. Huang and M. Muthukumar, J. Chem. Phys. 107, 5561 (1997).

[26] N. P. Rapapa and A. J. Bray, Phys. Rev. Lett. 83, 3856 (1999).

[27] F. Corberi, G. Gonnella, and A. Lamura, Phys. Rev. Lett. 81, 3852 (1998).

[28] J. Gunton, M. San Miguel, and P. Sahni, in Kinetics of first order phase transitions Vol. 8 of Phase Transitions and Critical Phenomena, eds. C. Domb and J. Lebowitz (Academic, London, 1983).

[29] J. Swift and P. C. Hohenberg, Phys. Rev. A 15, 319 (1977).

[30] K. R. Elder, J. Viñals, and M. Grant, Phys. Rev. Lett. 68, 3024 (1992); see also D. Boyer and J. Viñals, preprint cond-mat/0105543 and references therein.

[31] M. Laradji, H. Guo, M. Grant, and M. Zuckermann, J.Phys. A: Math. Gen 24, L629 (1991); J. Phys.: Condens. Matter 4, 6715 (1992).

[32] M. Bahiana and Y. Oono, Phys. Rev. A 41, 6763 (1990).

[33] G. Gonnella, E. Orlandini, and J. Yeomans, Phys. Rev. Lett. 78, 1695 (1997).

[34] See, e.g., A. Onuki, J. Phys.: Cond. Matt. 9, 6119 (1997).

[35] G. F. Mazenko and M. Zannetti, Phys. Rev. B 32, 4565 (1985).

[36] The proof is similar for NCOP; details will appear elsewhere.

[37] C. K. Chan, F. Perrot, and D. Beysens, Phys. Rev. A 43, 1826 (1991); K. Migler, C. Liu, and D. J. Pine, Macromolecules 29, 1422 (1996); F. Corberi, G. Gonnella, and A. Lamura, Phys. Rev. E 61, 6621 (2000).

[38] T. Ohta, H. Nozaki, and M. Doi, Phys. Lett. A 145, 304 (1990); J. Chem. Phys. 93, 2664 (1990).

[39] F. Corberi, G. Gonnella, and A. Lamura, Phys. Rev. Lett. 
83, 4057 (1999).

[40] A. H. Krall, J. V. Sengers, and K. Hamano, Phys. Rev. Lett. 69, 1963 (1992).

[41] A. Onuki, Phys. Rev. A 35, 5149 (1987).

[42] F. Corberi and U. Marini Bettolo Marconi, Europhys. Lett. 30, 349 (1995).

[43] A. Coniglio and M. Zannetti, Europhys. Lett. 10, 575 (1989).

[44] See, e.g., G. Mazenko in Formation and interaction of topological defects, eds. A.C.Davis and R. Brandenberger (Plenum. New York, 1995). 